\title{
Psychiatry in Sri Lanka
}

\author{
K. A. L. A. Kuruppuarachchi and R. R. Rajakaruna
}

Sri Lanka is a developing country situated in the Indian ocean with a population of about 18.5 million. Its education and health care services are free in the state sector. Psychiatric services are mainly confined to the units in the general hospitals and two large mental hospitals situated in Angoda and Mullertyawa (suburbs of Colombo) at the moment. However the institutional care is gradually changing and many professionals are aware of the importance of community care. There are a few reasonably organised community centres available at present.

Care for the mentally ill is mainly provided by psychiatrists. Many clinicians incorporate the family members in rehabilitation programmes and in some units family members are encouraged to visit and help the patients which may help to reduce the burden on the staff members at least to some extent and also it may be regarded as a 'community model'. Since the sub-specialities are poorly developed general adult psychiatrists are expected to deal with almost every clinical problem encountered in psychiatric practice, particularly in the provincial general hospitals. There are a few trained child psychiatrists, clinical psychologists and social workers who are also mainly confined to the major cities. Mentally ill patients are cared for mainly in the state sector while a small proportion are cared for in the private sector. However people from one geographical area are free to move to another area if the need arises as there are no clearly defined catchment areas for health care. The number of psychiatrists is grossly inadequate compared with Western standards.

There is a Mental Health Act in Sri Lanka. Since it is old and needs further revision a sub-committee has been appointed to look into this matter.

\section{Pattern of psychiatric morbidity}

Suicide rates and deliberate self-harm are major concerns in the mental health field. The suicide rate is remarkably high and Sri Lanka recorded the second highest suicide rate in the world during the period 1985-1990 (Abeysinghe, 1997). Agrochemical poisoning is a major cause of suicide. Pesticides are readily available as Sri Lanka is a farming country. The pattern of deliberate self-harm may be variable from one area to another. A prospective study done on deliberate self-harm at North Colombo General Hospital highlighted overdosing as a major method of attempted suicide (further details available from the author upon request).

Many clinicians often see post-traumatic stress disorders, adjustment disorders, bereavement reactions and other emotional disorders among servicemen and civilians. Child abuse, both emotional and sexual is also becoming more recognised. Fortunately, the relevant professionals and authorities are aware and concerned about these issues. Regular workshops and educational programmes are conducted to further educate the relevant professionals.

Alcohol-related problems and substance misuse are other major concerns. Locally distilled alcohol, kasippu, is a favourite cheap brand among the general population. Its alcohol concentration is grossly variable and there are many impurities. Cannabis (locally known as ganja) and heroin misuse is also encountered among the youth population - leading to many psychological and social problems. Some youths erroneously believe small quantities of ganja can increase appetite and improve strength and this can lead to the initiation of cannabis misuse.

As life expectancy is increasing (currently 69.5 years for men and $\mathbf{7 4 . 2}$ years for women) psychiatric problems of old age are also rising steadily. According to the population projections of the Ministry of Health the population above 65 years was $6.2 \%$ in 1995 and the projected figure for the year 2010 is $8.6 \%$ and for 2020 is $12.1 \%$ (Ministry of Health, Highways and Social Services, 1996).

Many people believe that the semen is a very special and a precious body fluid; so much so some equate one drop of semen to five drops of blood. Hence, some men are very anxious about nocturnal emissions and worried about masturbation, thinking that they may lose weight and strength and develop other bodily symptoms. Clinicians encounter a syndrome which is characterised by severe anxiety and hypochondriacal preoccupations associated with discharge of semen which is similar to dhat syndrome described in the DSM-IV (American Psychiatric Association, 1994). This is known as sukra prameha locally.

Somatic symptoms (e.g. burning sensation of the abdomen, head or the whole body) in depressive illness is a common presentation. 
mainly in the elderly. Another interesting observation is that the clinicians still see full blown catatonia, due to depressive illness, schizophrenia, or organic conditions, for example viral encephalitis. The reasons may be delay in treatment, presence of a variety of viruses causing encephalitis or other unknown causes. Research work is needed in this area.

\section{Traditional practices and newer therapies}

Many people, depending on their level of education and awareness, attribute supernatural or spiritual influence in the causation of mental illness. Traditional forms of healing may vary from applying a chanted or charmed thread to exorcism. In exorcism the basic assumption is that mental illnesses are caused by supernatural influences and the healer performs a variety of rituals including drumming and dancing in order to get rid of the suspected evil spirit from the patient's body or soul (see Figs 1 and 2). Some believe that the influence of the spirits of dead relatives or ghosts can cause mental illness.

Significant numbers of patients or their relatives perform a variety of religious activities and rituals believing that they can enhance the healing process. A survey carried out in a provincial general hospital by K.A.L.A.K. (further details available from the author upon request) showed that up to $80 \%$ of the patients with puerperal mental disorders sought help from some form of religious or traditional healing method (at least applying a chanted thread) in addition to Western treatment. The common belief is that it will help the recovery process and protect the patient from evil spirits.

Selective serotonin reuptake inhibitors (fluoxetine and paroxetine) have been avallable over the last few years, mainly in the private sector. Clinicians have to think twice before prescribing these newer drugs as poor people find them difficult to afford because of the relatively high cost. Clozapine and risperidone are also available. However, also due to high cost these drugs are prescribed in a limited way. Clinicians incorporate principles of cognitive-behavioural therapy in their patient management, particularly for anxiety disorders and depressive illness, as it is an affordable form of psychotherapy.

\section{Psychiatric training and future needs}

Many medical schools have recognised the importance of incorporating psychiatry into their undergraduate curricula. Some medical schools have made a pass grade in psychiatry compulsory at the final year assessment.

Postgraduate psychiatric training consists of a five-year compulsory training period. The

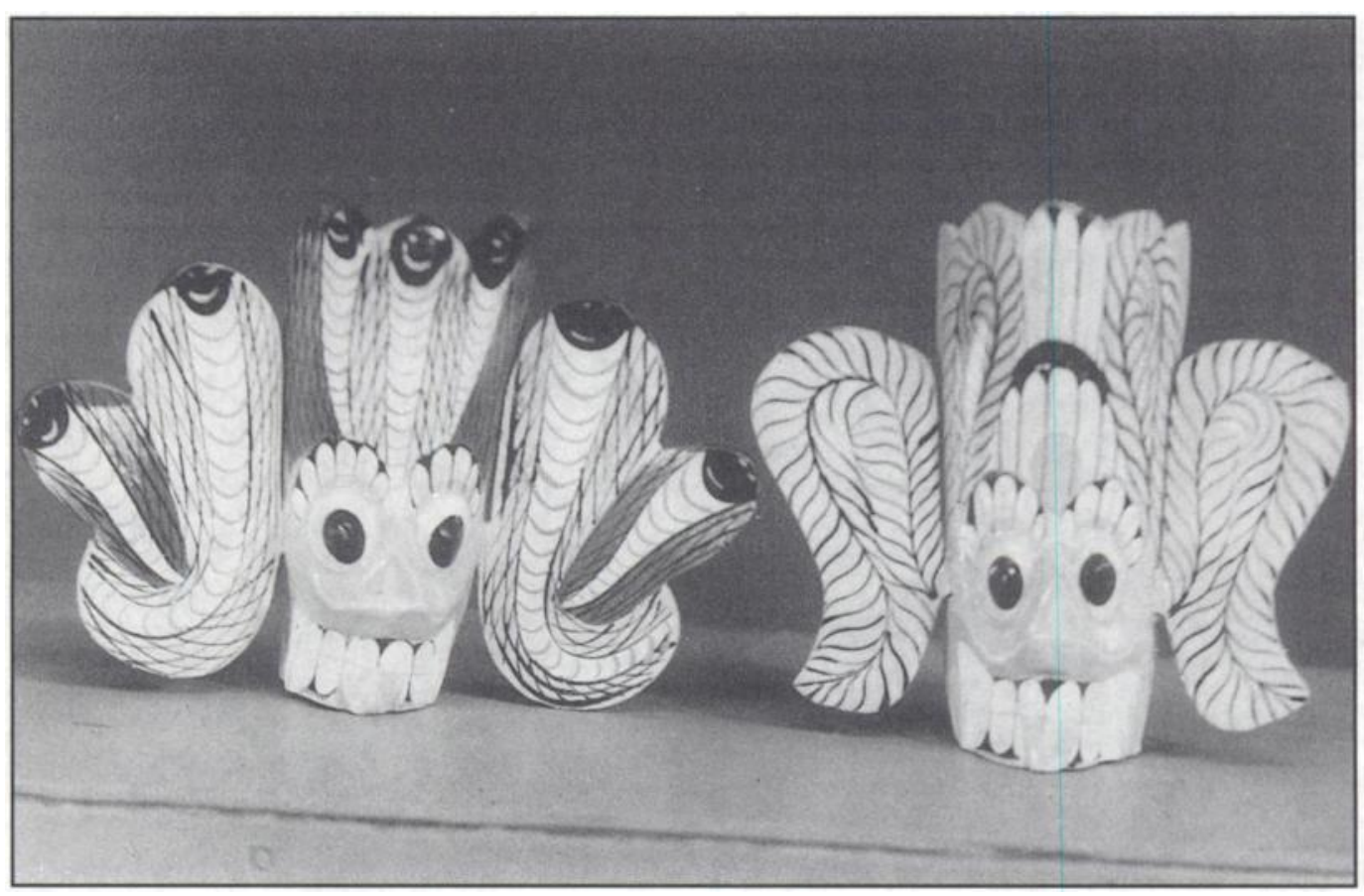

Fig. 1. Examples of the masks worn by exorcists. 


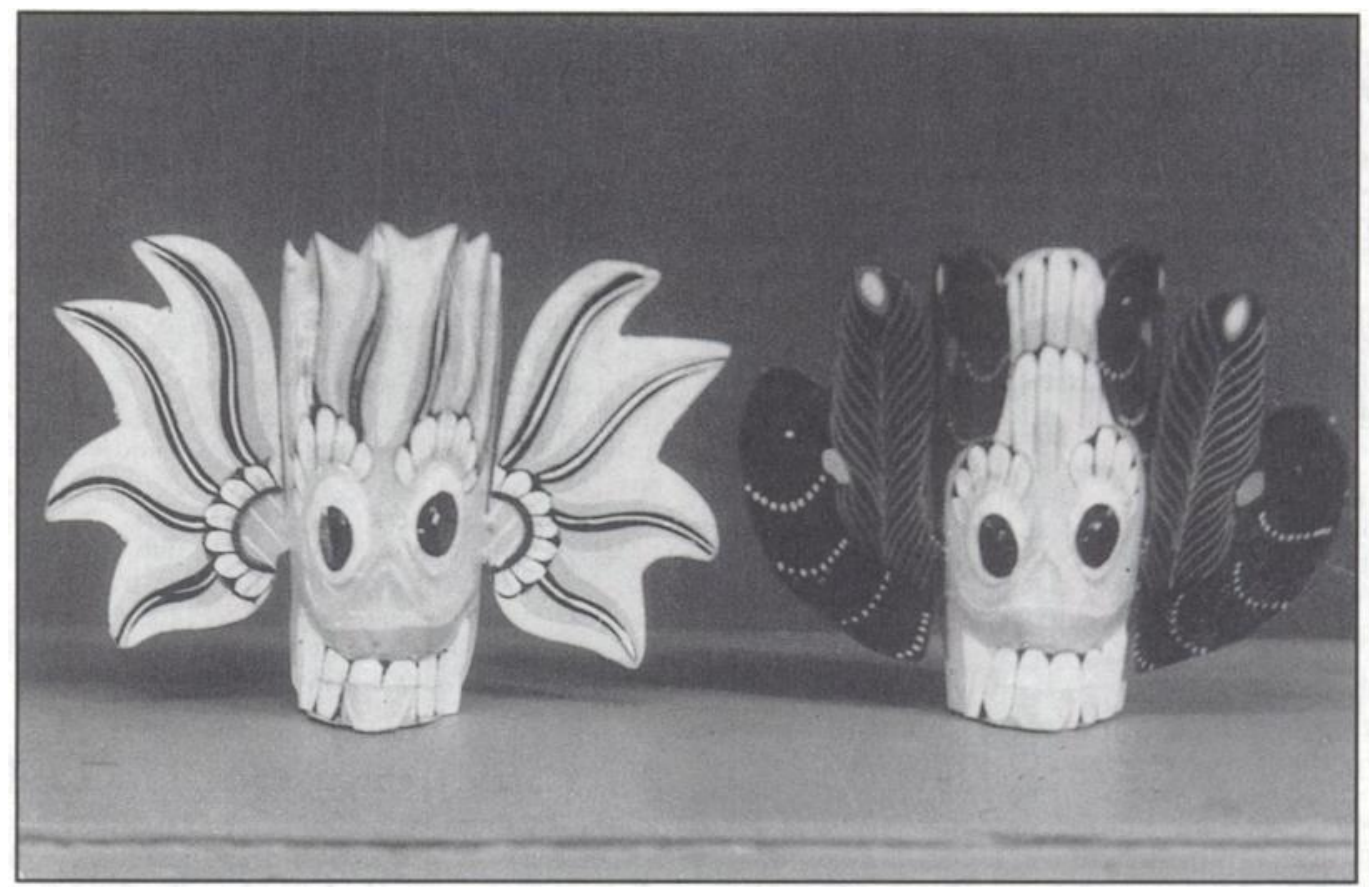

Fig. 2. A variety of masks are used, depending on the illness.

trainees are eligible to enter the training programme after successful completion of the MD Part 1 Examination (knowledge of the basic sciences relevant to psychiatry and relevant general medicine and neurology are assessed in the Part 1). In the MD Part 2 Examination knowledge of the whole range of theoretical concepts and clinical skills are assessed. An external examiner, often from the UK, participates in the MD Part 2 assessment. After successful completion of the MD Part 2, trainees are expected to undergo a further year training in Sri Lanka and at least one year of training abroad, preferably in the UK or Australia. Many trainees get placements through the Overseas Doctors Training Scheme (UK) dual sponsorship programme. The majority feel this component of the training programme is of great benefit in order to widen the perspectives and to gather more skills. After completion of all the components of the training programme (including a dissertation on a topic relevant to psychiatry) successfully, the trainee is board certified as a specialist in psychiatry.

We need to increase the awareness of mental health ailments among the people in order to minimise the stigma and to allow them to be identified early. Rehabilitation of people with mental illness may be improved and long-term institutional care minimised by reinforcing community-oriented approaches to psychiatric disorders. As the extended family system is gradually changing (particularly in the urban settings) one has to seriously consider psychiatric problems encountered in old age. The other important area that needs to be improved is child psychiatry - as behavioural problems and emotional disorders encountered in children are becoming more recognised.

\section{References}

ABEYSINGHE, D. R. R. (1997) Suicide in Sri Lanka. Kandy Medical Journal, 6, 32-34.

AMERICAN PSYCHIATRIC ASSOCIATION (1994) Dlagnostic and Statistical Manual of Mental Disorders. (4th edn) DSMIV. Washington. DC: American Psychiatric Association. Ministry OF HEALTH, HighwAYS AND SOCIAL SERVICES (1996) Population Statistics of Sri Lanka. Colombo: Population Information Centre.

*K. A. L. A. Kuruppuarachchi, Senior Lecturer in Psychiatry, and R. R. Rajakaruna, Medical Officer, Department of Psychiatry, Faculty of Medicine, University of Kelaniya, Ragama, Sri Lanka

*Correspondence 